\title{
Avaliação da relação entre variação de espessura da placa de concreto e presença de defeitos em pavimentos de concreto
}

\author{
Deividi da Silva Pereira' ${ }^{1}$, Lúcio Salles de Salles², Flavia Campos Macedo Britto ${ }^{3}$, \\ Diego Sousa Garcia Sanz ${ }^{4}$, Lev Khazanovich ${ }^{5}$, Kyle Hoegh ${ }^{6}$
}

1Departamento de Engenharia de Transportes, Universidade Federal de Santa Maria, Santa Maria, dsp@ufsm.br 2Departamento de Engenharia Civil, Universidade Federal de São Carlos, lucio.salles@ufscar.br 3Engenheira Civil, Bureau Veritas Brasil, flavia.m.britto@gmail.com ${ }^{4}$ Engenheiro Civil, Management Solutions, diegosgsanz@gmail.com ${ }^{5}$ Department of Civil and Environmental Engineering, University of Pittsburgh, lev.k@pitt.edu ${ }^{6}$ Research Scientist 3, Minnesota Department of Transportation, kyle.hoegh@state.mn.us

\section{Recebido:}

29 de julho de 2017

Aceito para publicação:

28 de março de 2018

Publicado:

31 de dezembro de 2018

Editor de área:

Francisco Thiago Aragão

\section{Palavras-chaves:}

Pavimento de concreto;

Espessura de placa;

Defeitos;

Variabilidade de espessura;

Uniformidade de construção.

\section{Keywords:}

Concrete pavement;

Slab thickness;

Distresses;

Thickness;

Variability;

Construction uniformity.

DOI:10.14295/transportes.v26i4.1441

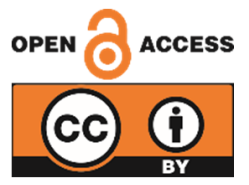

\section{RESUMO}

A espessura da placa de concreto é a principal incógnita de métodos de dimensionamento de pavimentos de concreto. Pressupõe-se que para condições semelhantes, pavimentos com placas espessas apresentem melhor desempenho do que aqueles com placas delgadas. Entretanto, o efeito da variabilidade da espessura final é pouco discutido. Neste artigo, foram relacionadas informações de avaliações acerca da variabilidade de espessura de pavimentos de concreto com mapas de defeitos na superfície das mesmas placas. Observou-se uma grande variação da espessura do pavimento em ambas as direções. No entanto, o desempenho dos pavimentos (número de defeitos) não depende unicamente da relação entre a espessura de campo e a de projeto. Seções com placas mais espessas do que a espessura de projeto não obtiveram, necessariamente, os melhores desempenhos. Isso se torna importante, considerando que construtoras tendem a construir placas ligeiramente mais espessas, destinadas a evitar deduções de contrato e penalidades. Por outro lado, seções com pequenas deficiências, mas com menor variação de espessura apresentaram um menor número de defeitos, sugerindo o desvio-padrão da espessura (uniformidade de construção) como um parâmetro relevante para o controle de qualidade.

\section{ABSTRACT}

Concrete slab thickness is the main output of any concrete pavement design method. It is assumed that, for similar conditions, concrete pavements with thicker slabs would present a better performance than ones with thinner slabs. However, the effect of field slab thickness variability is rarely analyzed. In the proposed paper, a comprehensive evaluation of slab thickness variability of several concrete pavements was related to surface distress maps in the same slabs. The results show great slab thickness variation in both directions. However, the concrete pavement performance (number of distresses) does not depend solely on the relationship between the field slab thickness and the design thickness. Sections with slabs thicker than the design thickness did not necessarily presented better performance. This becomes important, especially considering that contractors tend to build slightly thicker slabs in order to avoid contract deductions and penalties. On the other hand, sections with small thickness deficiencies but with smaller thickness variation presented fewer distresses suggesting the thickness standard deviation (construction uniformity) as a relevant parameter for quality control.

\section{INTRODUÇÃO}

Pavimentos de Concreto de Cimento Portland (CCP) são caracterizados pela sua elevada rigidez 
que permite a transferência dos carregamentos oriundos do tráfego de maneira bastante atenuada para as camadas subjacentes, inclusive para o subleito. As placas de concreto configuramse em uma alternativa técnica importante e viável, sobretudo para fluxo canalizado e de elevados volumes/cargas de tráfego. Entretanto, de maneira similar a todas as alternativas de pavimentação, para os pavimentos de concreto, vários aspectos de projeto e construtivos afetam o comportamento e o desempenho do pavimento.

De acordo com NCHRP (2004), existem algumas características de projetos, incluindo propriedades do material, barras de ligação e de transferência, espaçamento de juntas, tipo de base, drenagem e espessura que interferem decisivamente no comportamento da estrutura viária. Tecnicamente, um dos parâmetros mais sensível em relação ao desempenho do pavimento é a espessura da placa de CCP que deve ser cuidadosamente dimensionada e controlada durante a construção. 0 conhecimento tácito da pavimentação em concreto diz que a deficiência de espessura pode causar consideráveis falhas construtivas sendo, por outro lado, o excesso de espessura um desperdício financeiro e de material. Por sua importância, falhas no controle da espessura do CCP durante a execução das placas podem ensejar penalizações por conta de espessuras insuficientes. O controle da espessura de placas de CCP no estado de Minnesota (MNDOT, 2015) é realizado a partir de extrações de corpos-de-prova (CP) por sonda rotativa a cada 1000 pés $(305 \mathrm{~m})$ enquanto que no Brasil, o Departamento Nacional de Infraestrutura de Transportes (DNIT, 2004) regula um controle geométrico de nivelamento de 20 em $20 \mathrm{~m} \mathrm{du}$ rante a construção do pavimento. Com isso, diversos estudos norte-americanos evidenciaram uma tendência dos construtores de executar placas ligeiramente mais espessas do que o projeto, evitando, assim, penalidades devido à falta de espessura (STUBSTAD et al, 2002; JIANG, et al, 2003; KIM e MCCULLOUGH, 2002).

Considerando a espessura do pavimento como um importante critério de projeto, este artigo apresenta uma análise da variação de espessura de seções de pavimento de CCP mensurada com um equipamento ultrassônico não destrutivo. A variação da espessura foi relacionada com a presença de defeitos na superfície do pavimento obtida através de tecnologia de imagens continuas do pavimento. Esta pesquisa foi desenvolvida pelo Departamento de Transportes do Estado de Minnesota (MnDOT) e pela Universidade de Minnesota (UofM), ambos nos Estados Unidos, para investigar uma possível correlação entre a variação de espessura e defeitos ao longo do pavimento.

\section{METODOLOGIA DE COLETAS DE DADOS}

Com a finalidade de investigar o efeito da variação de espessura na ocorrência de defeitos ao longo do pavimento, foram selecionados três segmentos de rodovias existentes no estado de Minnesota, no norte dos Estados Unidos, e que estavam prestes a serem restauradas. Eram, portanto, vias de CCP que apresentavam defeitos em suas placas. Estes trechos estão indicados na Tabela 1, com as devidas informações de projeto. Todos os pavimentos investigados foram de concreto continuamente armado (PCCA), de emprego frequente em Minnesota, com bases granulares de diferentes espessuras. 
Tabela 1: Especificações de Projeto das seções analisadas

\begin{tabular}{lllllll}
\hline & \multicolumn{9}{c}{ Dimensões da placa } \\
\hline Seção & $\begin{array}{l}\text { Tipo de } \\
\text { Pavimento de } \\
\text { CCP }\end{array}$ & $\begin{array}{l}\text { Espessura } \\
\text { de Projeto }\end{array}$ & Largura x comprimento & Base & $\begin{array}{l}\text { Espessura } \\
\text { da Base }\end{array}$ & $\begin{array}{l}\text { Ano de } \\
\text { Construção }\end{array}$ \\
\hline & & $(\mathrm{mm})$ & $(\mathrm{m})$ & Granular & 127 & 1998 \\
\hline Rodovia 60 (Rod-60) & PCCA & 203,2 & $3,66 \times 8,23$ & Granular & 330,2 & 1973 \\
Rodovia 100 (Rod-100) & PCCA & 228,6 & $3,66 \times 8,23$ & Granular & 76,2 & 1990 \\
Interestadual 394 (I-394) & PCCA & 254 & $3,66 \times 8,23$ & &
\end{tabular}

\subsection{Investigação de Defeitos e de variação de espessura}

Todos os anos, o departamento responsável pela Gerência de Pavimentos do MnDOT realiza um cadastro fotográfico alusivo à condição do pavimento através de um veículo de inspeção digital como mostrado na Figura 1a. (MNDOT, 2006). 0 software chamado Videolog foi utilizado para processar os dados e realizar a investigação de defeitos. 0 Videolog é composto de quatro janelas (Vista Frontal, Vista da Superfície, Janela de Localização e Janela de Controle), conforme Figura 1b, e permite a localização precisa dos defeitos com precisão, devido a uma ferramenta de medição no software, vinculada ao estaqueamento da rodovia.

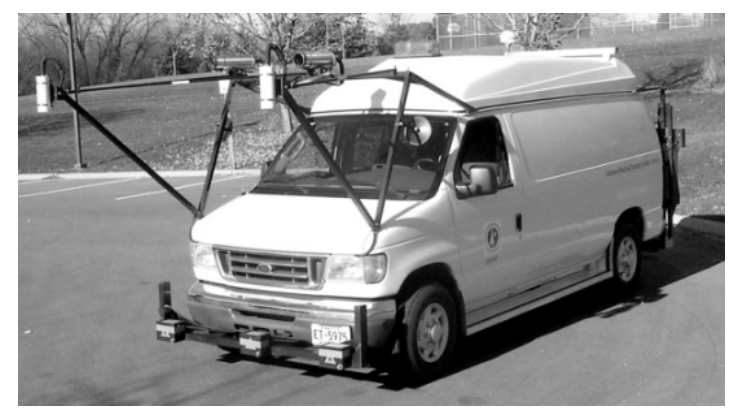

(a)

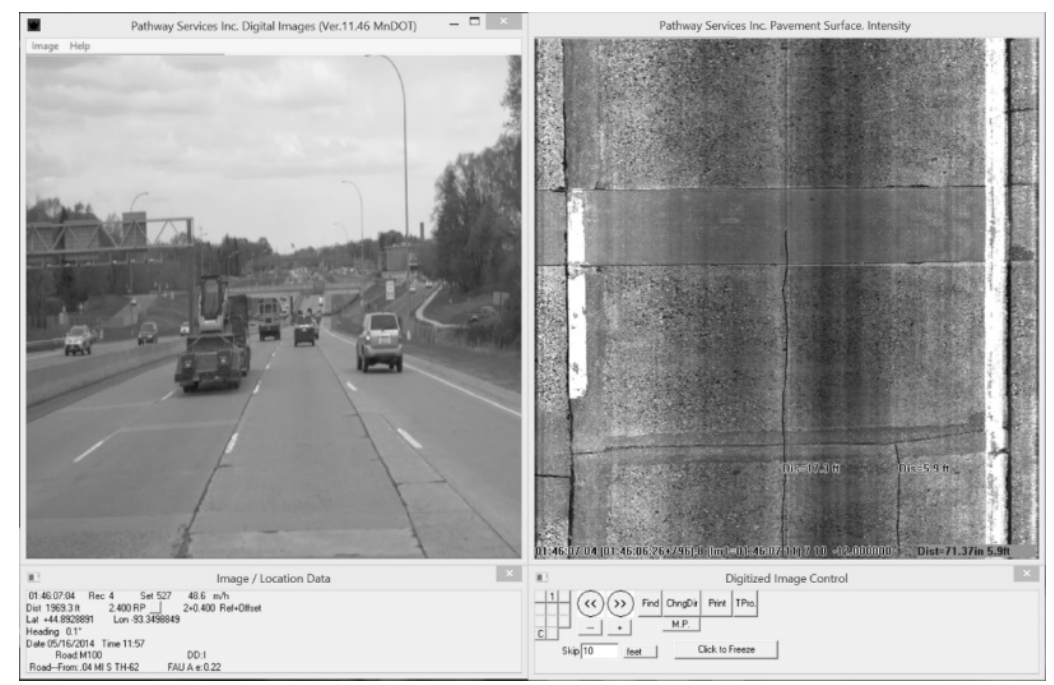

(b)

Figura 1: Procedimento de avalição visual de defeitos - (a) veículo e (b) software utilizados

A metodologia empregada para a investigação de defeitos neste estudo foi baseada na tipificação de defeitos definidos em MnDOT (2011) enquanto que os métodos de localização de defeitos fazem referência à FHWA (2003). Desta maneira, os defeitos foram classificados em sete tipos diferentes, a saber: \#1 esborcinamento de juntas; \#2 placa fissurada; \#3 placa fissurada 
em mais de uma direção (Figura 2a); \#4 escalonamento em placa fissurada; \#5 placa com remendo asfáltico; \#6 remendo de CCP em placa (Figura 2b); e \#7 delaminação (D-Cracking). Os defeitos foram registrados em cinco posições transversais na placa, de acordo com a Figura 3, sendo a primeira posição junto à borda longitudinal externa do pavimento (junto ao acostamento) e a quinta próxima à junta longitudinal limítrofe a outra faixa de rolamento.

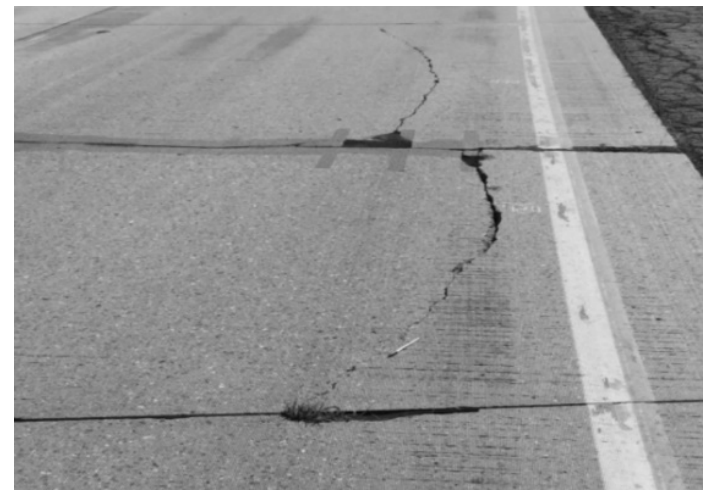

(a)

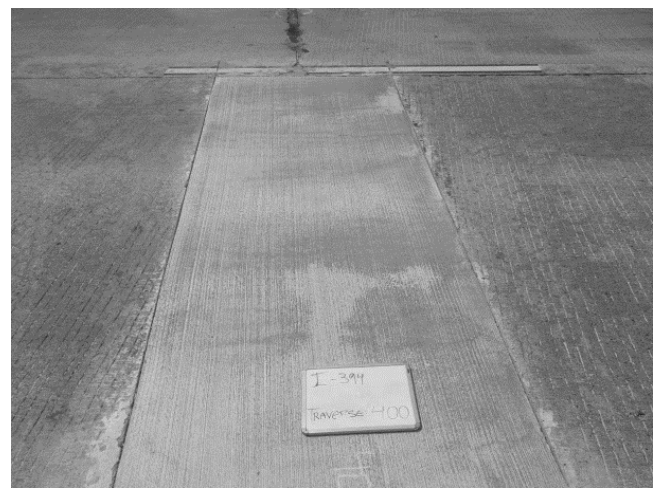

(b)

Figura 2: (a) Placa fissura em mais de uma direção na seção Rodovia 60 e (b) remendo de placa na Interestadual 394

\section{Duplas de leitura do MIRA}

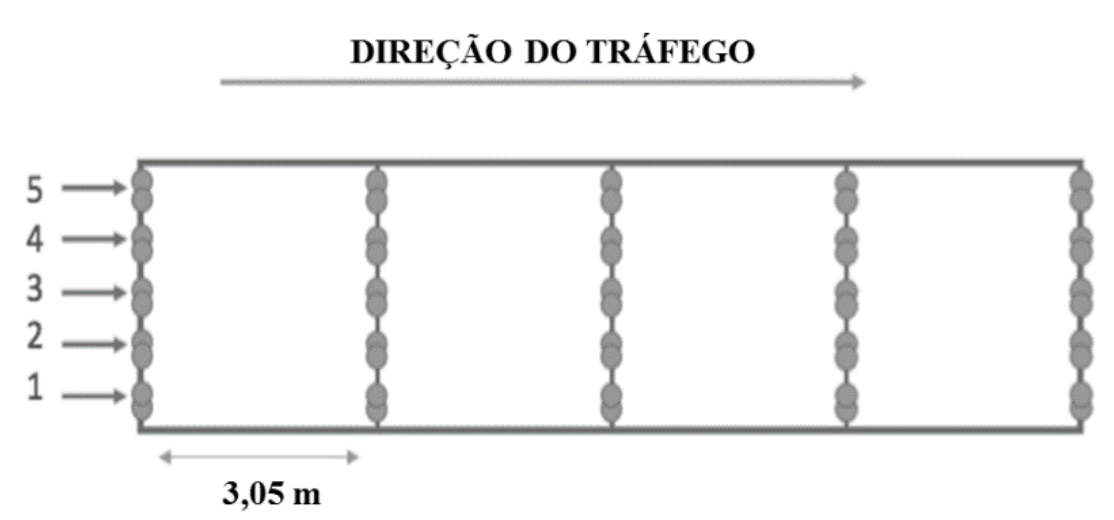

Figura 3: Posição de leitura de espessura e de localização de defeitos

\subsection{Investigação de Espessuras}

O método de investigação de espessura utilizado nesta pesquisa foi resultado de diferentes padrões testados previamente, especialmente naquele desenvolvido por Vancura et al. (2013). 0 método consistiu em 10 medidas na transversal a cada 3,05m (10 pés) conforme apresentado na Figura 3. As medidas foram tomadas em cinco duplas de leituras nas cinco posições da seção transversal. Desse modo, os resultados de espessura de cada dupla são comparados como forma de verificação do levantamento. Se as espessuras aferidas por cada par de levantamentos diferirem mais do que $6,35 \mathrm{~mm}$, a medida era considerada não conclusiva e eliminada da análise final. Como o levantamento de defeitos foi realizado nas mesmas posições das leituras de espessura, foi possível correlacionar, diretamente, a espessura avaliada e o defeito eventualmente existente na periferia destes pontos.

A espessura da placa de concreto foi mensurada através de um equipamento de tomografia ultrassônica capaz de gerar uma imagem ultrassônica do interior da placa de concreto devido a 40 transmissores e receptores com ponto de contato seco na parte inferior do dispositivo, como mostrado na Figura 4. Estes transdutores são fixados em uma matriz com 10 canais (colunas) 
de quatro transdutores transversais, com uma visualização de tamanho de 400 por 50 mm. No total, cada leitura deste equipamento coleta sinais de 45 transmissores e receptores em pares (Figura 4), gerando uma infinidade de sinais, promovendo elevada confiabilidade quando aplicado a materiais heterogêneos, tal como o CCP. (HOEGH et al, 2011; VANCURA et al, 2013).

As ondas de baixa frequência geradas pelos transdutores distribuem-se através do pavimento de concreto sendo refletidas em um ponto de descontinuidade acústica. As posições de alta intensidade são descritas como uma mudança na impedância acústica, que pode ser uma interface de duas camadas, danos ao concreto, delaminação ou até barras da armadura de ligação ou de transferência (HOEGH et al, 2012; HOEGH et al, 2013; SALLES et al 2017).
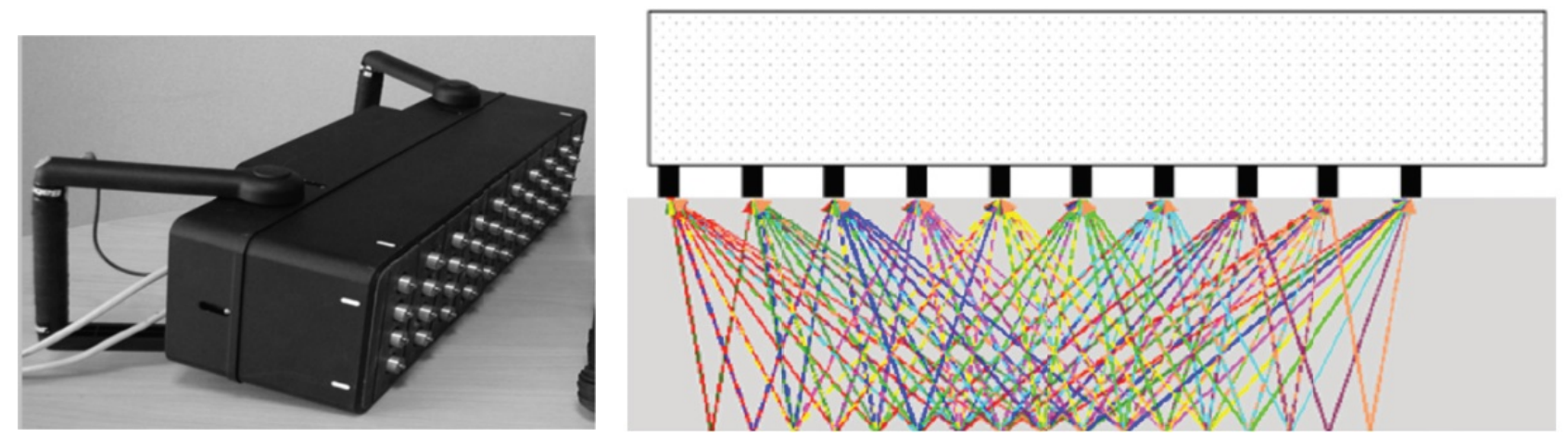

Figura 4: Dispositivo ultrassônico MIRA (VANCURA et al, 2013)

Em uma pesquisa proposta por Vancura et al. (2013), em que o objetivo principal foi avaliar se os corpos de prova extraídos segundo às diretrizes normativas do MnDOT representariam fielmente a variação da espessura do pavimento de concreto, comparou-se as informações de espessura obtidas a partir da tomografia ultrassônica (MIRA) e àquelas oriundas da perfuração de corpos de prova. Neste trabalho, a precisão das leituras realizadas pelo MIRA foi validada com grande sucesso $\left(R^{2}=0.9968\right)$. Ótimas correlações entre os resultados do MIRA e CPs foram também encontrados em Hoegh et al. (2012) e Edwards (2012).

\section{RESULTADOS E ANÁLISE}

Para elaborar uma comparação entre a variação de espessura e defeitos observados, foram executados 4.190 pares de leituras, perfazendo a avaliação de mais de $2.500 \mathrm{~m}$ de faixa de rolagem de pavimentos de CCP. Cada rodovia analisada nesse estudo foi dividida em segmentos de 152 m (500 pés) para realizar comparações mais precisas entre o número de defeitos observados e a variação da espessura em cada seção.

Devido ao volumoso número de dados coletados, apresenta-se a Tabela 2 que sintetiza os tipos de defeitos encontrados em cada seção. De maneira análoga, um resumo com os resultados estatísticos das espessuras dos três pavimentos avaliados é apresentado na Tabela 3. Desenvolveu-se, também, uma análise estatística de cada posição transversal (Tabela 4), necessária à avaliação das posições com maiores espessura nominais bem como sua maior dispersão.

Uma vez que os segmentos avaliados foram inaugurados em diferentes anos, sendo submetidos a distintos perfis de tráfego, carregamentos e condições climáticas, além das diferentes espessuras de placa e base, optou-se pela análise das seções de forma individual. 
Tabela 2: Resumo quantitativo dos defeitos

\begin{tabular}{|c|c|c|c|c|c|c|c|c|c|}
\hline \multicolumn{10}{|c|}{ Defeitos* } \\
\hline Seção & Segmento & $\begin{array}{l}\text { Total de } \\
\text { defeitos }\end{array}$ & 1 & 2 & 3 & 4 & 5 & 6 & 7 \\
\hline Rod-60 Oeste & 10 (0ft - 500ft) & 7 & 1 & 5 & - & - & - & 1 & - \\
\hline Rod-60 Oeste & $20(500 f t-1000 f t)$ & 17 & - & 16 & - & - & - & 1 & - \\
\hline Rod-60 Oeste & 30 (1000ft - 1500ft) & 26 & - & 21 & - & - & - & 5 & - \\
\hline Rod-60 Oeste & 40 (1500ft - 2000ft) & 24 & - & 15 & 3 & - & - & 6 & - \\
\hline Rod-60 Oeste & 50 (2000ft - 2500ft) & 21 & - & 17 & 1 & 1 & - & 2 & - \\
\hline Rod-60 Oeste & $60(2500 f t-3000 f t)$ & 18 & - & 17 & - & - & - & 1 & - \\
\hline Rod-60 Leste & 70 (3000ft - 3500ft) & 26 & - & 18 & 4 & - & - & 4 & - \\
\hline Rod-60 Leste & 80 (3500ft - 4000ft) & 25 & - & 16 & 6 & - & - & 3 & - \\
\hline Rod-60 Leste & 90 (4000ft - 4500ft) & 12 & - & 9 & 1 & - & - & 2 & - \\
\hline Rod-60 Leste & $10^{\circ}$ (4500ft - 5000ft) & 7 & - & - & 4 & - & - & 3 & - \\
\hline Rod-100 & 10 (0ft - 500ft) & 4 & - & 3 & - & - & - & 1 & - \\
\hline Rod-100 & $20(500 f t-1000 f t)$ & 6 & - & 4 & - & - & - & 2 & - \\
\hline Rod-100 & $30(1000 f t-1380 f t)$ & 1 & - & - & - & - & - & 1 & - \\
\hline $1-394$ & $10(0 f t-500 f t)$ & 15 & - & 3 & - & - & - & 12 & - \\
\hline I-394 & 20 (500ft - 1000ft) & 14 & - & 2 & 1 & - & - & 10 & - \\
\hline I-394 & 30 (1000ft - 1500ft) & 3 & - & 1 & - & - & - & 2 & - \\
\hline |-394 & 40 (1500ft - 2000ft) & 7 & - & 1 & - & - & - & 6 & - \\
\hline
\end{tabular}

*Defeitos:

1.esborcinamento de juntas; 2. placa fissurada; 3 placa fissurada em mais de uma direção;

4. escalonamento em placa fissurada; 5. placa com remendo asfáltico; 6. remendo de CCP em placa

7. delaminação (D-Cracking)

Tabela 3: Síntese dos resultados estatísticos de espessura

\begin{tabular}{|c|c|c|c|c|c|c|}
\hline Seção & Segmento & $\begin{array}{l}\text { Total de } \\
\text { defeitos }\end{array}$ & $\begin{array}{l}\text { Média de } \\
\text { Esp. }(\mathrm{mm})\end{array}$ & $\begin{array}{l}\text { Desvio } \\
\text { Padrão }\end{array}$ & $\begin{array}{l}\text { Máxima } \\
\text { Esp. }(\mathrm{mm})\end{array}$ & $\begin{array}{l}\text { Mínimo } \\
\text { Esp. (mm) }\end{array}$ \\
\hline Rod-60 Oeste & $10(0 \mathrm{ft}-500 \mathrm{ft})$ & 7 & 187 & 8,3 & 218,5 & 169 \\
\hline Rod-60 Oeste & $20(500 \mathrm{ft}-1000 \mathrm{ft})$ & 17 & 199,7 & 7,8 & 224 & 183,5 \\
\hline Rod-60 Oeste & 30 (1000ft - 1500ft) & 26 & 206,1 & 9,6 & 234 & 186,5 \\
\hline Rod-60 Oeste & 4o (1500ft - 2000ft) & 24 & 201,9 & 8,6 & 223,5 & 180,5 \\
\hline Rod-60 Oeste & 50 (2000ft - 2500ft) & 21 & 205,6 & 9 & 229 & 184,5 \\
\hline Rod-60 Oeste & $60(2500 \mathrm{ft}-3000 \mathrm{ft})$ & 18 & 193,1 & 8,4 & 215 & 174 \\
\hline Rod-60 Leste & 70 (0ft - 500ft) & 26 & 199,8 & 8,8 & 218,5 & 177,5 \\
\hline Rod-60 Leste & $80(500 \mathrm{ft}-1000 \mathrm{ft})$ & 25 & 198,9 & 11,7 & 225 & 176 \\
\hline Rod-60 Leste & 9o (1000ft - 1500ft) & 12 & 193,9 & 8 & 214 & 175,5 \\
\hline Rod-60 Leste & 10 o Seção (1500ft - 2000ft) & 7 & 187,4 & 6,9 & 213 & 171,5 \\
\hline Rod-100 & $10(0 \mathrm{ft}-500 \mathrm{ft})$ & 4 & 221,7 & 10,8 & 255 & 189,5 \\
\hline Rod-100 & $20(500 \mathrm{ft}-1000 \mathrm{ft})$ & 6 & 217,1 & 10 & 246 & 194,5 \\
\hline Rod-100 & 3ㅇ (1000ft - 1380ft) & 1 & 219,8 & 7,7 & 238 & 200,5 \\
\hline $1-394$ & 1ㅇ Seção (0ft - 500ft) & 15 & 261,7 & 7,8 & 283 & 245 \\
\hline I-394 & 2o Seção (500ft - 1000ft) & 14 & 258 & 8,1 & 277 & 239 \\
\hline I-394 & 3o Seção (1000ft - 1500ft) & 3 & 253,9 & 7,6 & 277,5 & 231,5 \\
\hline I-394 & 4o Seção (1500ft - 2000ft) & 7 & 260,7 & 7,4 & 279 & 240,5 \\
\hline
\end{tabular}

Tabela 4: Resumo de espessuras por posições transversais

\begin{tabular}{lllllll}
\hline \multicolumn{7}{c}{ Posição Transversal } \\
& & $\mathbf{1}$ & $\mathbf{2}$ & $\mathbf{3}$ & $\mathbf{4}$ & $\mathbf{5}$ \\
(Borda Ext.) & (Trilha Ext.) & $\begin{array}{l}\text { (Centro) } \\
\text { (Trilha Int.) }\end{array}$ & (Borda Int.) \\
\cline { 3 - 7 } Rod-60 & Média da Espessura (mm) & 194,4 & 194,5 & 197,4 & 200 & 200,6 \\
& Desvio Padrão (mm) & 7,2 & 7,9 & 9,9 & 12,1 & 14,2 \\
\hline \multirow{2}{*}{ Rod-100 } & Média da Espessura (mm) & 216,5 & 221,1 & 218,5 & 219,9 & 221,4 \\
& Desvio Padrão (mm) & 9,9 & 9,6 & 10 & 9,5 & 10 \\
\multirow{2}{*}{ I-394 } & Média da Espessura (mm) & 252,9 & 256,1 & 263,9 & 259,6 & 261,7 \\
& Desvio Padrão (mm) & 7,9 & 7,3 & 6,7 & 7 & 7,5 \\
\hline
\end{tabular}




\subsection{Rodovia 60}

Esta rodovia, diferente das demais, teve os levantamentos realizados em duas faixas de rolagem, nas faixas mais carregadas (externa) de cada uma das duas pistas que a constituem, totalizando $914,4 \mathrm{~m}$ em um sentido e 609,6 m no sentido oposto. Entretanto, neste trabalho, os dois segmentos foram unidos para facilitar as análises e porque o projeto estrutural do pavimento e a condição de tráfego atuante, em ambos os sentidos, eram similares.

Subtraindo-se a mínima espessura medida deste trecho (169 mm - vide Tabela 3) da espessura de projeto (203,2 mm - Tabela 1), tem-se uma diferença de 34,2 mm, que representa uma disparidade de valores que excederia o limite apontado pelo MnDOT (2015). A área de abrangência da referida diferença de espessura, 34,2 mm, implicaria em uma das duas possibilidades, conforme decisão da fiscalização:

1. Remoção e substituição das placas naquela região, sem custos para o departamento; ou

2. empreiteiro não receberia pelo serviço realizado incidindo, adicionalmente, uma multa de US $\$ 25,00 / \mathrm{m}^{2}$ relativo à região com diferença superior a $25 \mathrm{~mm}$ entre a espessura de projeto e a existente.

A Figura 5 possibilita a percepção da existência de um grande número de pontos com espessura de placa abaixo da daquela de projeto, o que resulta em uma espessura média do trecho abaixo da linha referente ao projetado. Esta diferença entre a espessura média de todo o segmento $(197,4 \mathrm{~mm})$ e a de projeto $(203.2 \mathrm{~mm})$ é de $5,8 \mathrm{~mm}$, o que acarretaria em uma dedução monetária de US\$ 0,50 por metro quadrado, caso a avaliação fosse determinada com base na média geral, o que de fato não traduz a orientação normativa encontrada em MnDOT (2015). Ainda, percebe-se uma grande dispersão de pontos (representativos de espessuras avaliadas pelo equipamento ultrassônico) em relação à espessura média ou, mesmo, àquela de projeto. Esta dispersão é maior que a encontrada, por exemplo, na Rodovia 100 que será melhor discutida na sequência (vide Figura 6).

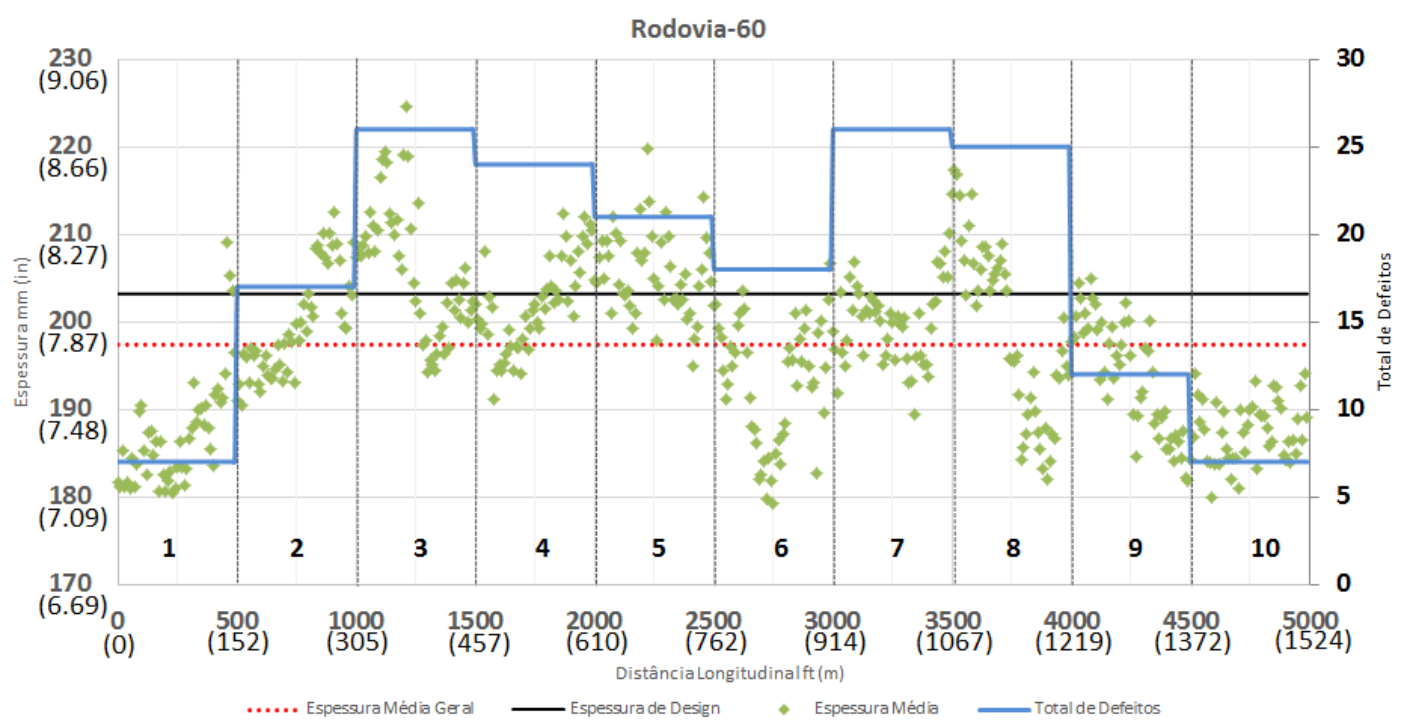

Figura 5: Variação longitudinal da espessura versus defeitos (Rodovia 60)

Um estudo individual de cada segmento mostra que o terceiro e o quinto segmentos possuem espessuras médias ligeiramente maiores que a espessura de projeto (vide Tabela 3); logo, seria 
presumível que estes segmentos teriam um comportamento melhor (menos defeitos). Entretanto, a Figura 5 exibe claramente que estes segmentos não correspondem àqueles com o menor número de defeitos. A seção 8 é a que apresenta o maior desvio padrão de espessura (11,7mm), assim como elevado número de defeitos. Em contrapartida, a seção 10 apresenta o menor número de defeitos e um menor desvio padrão $(6,9 \mathrm{~mm})$.

A análise transversal da rodovia 60 (Tabela 4) mostra a posição 5 (borda longitudinal interna) com o maior desvio padrão de espessura e maior espessura média, bem como a posição 1 (borda externa) com o menor desvio padrão e menor média.

\subsection{Rodovia 100}

As medidas de espessuras e o número de defeitos são apresentados na Figura 6. Nota-se, claramente, uma maior concentração de pontos abaixo da linha de espessura de projeto, resultando em uma espessura média geral de toda a via avaliada inferior à de projeto, conforme visto na Tabela 3.

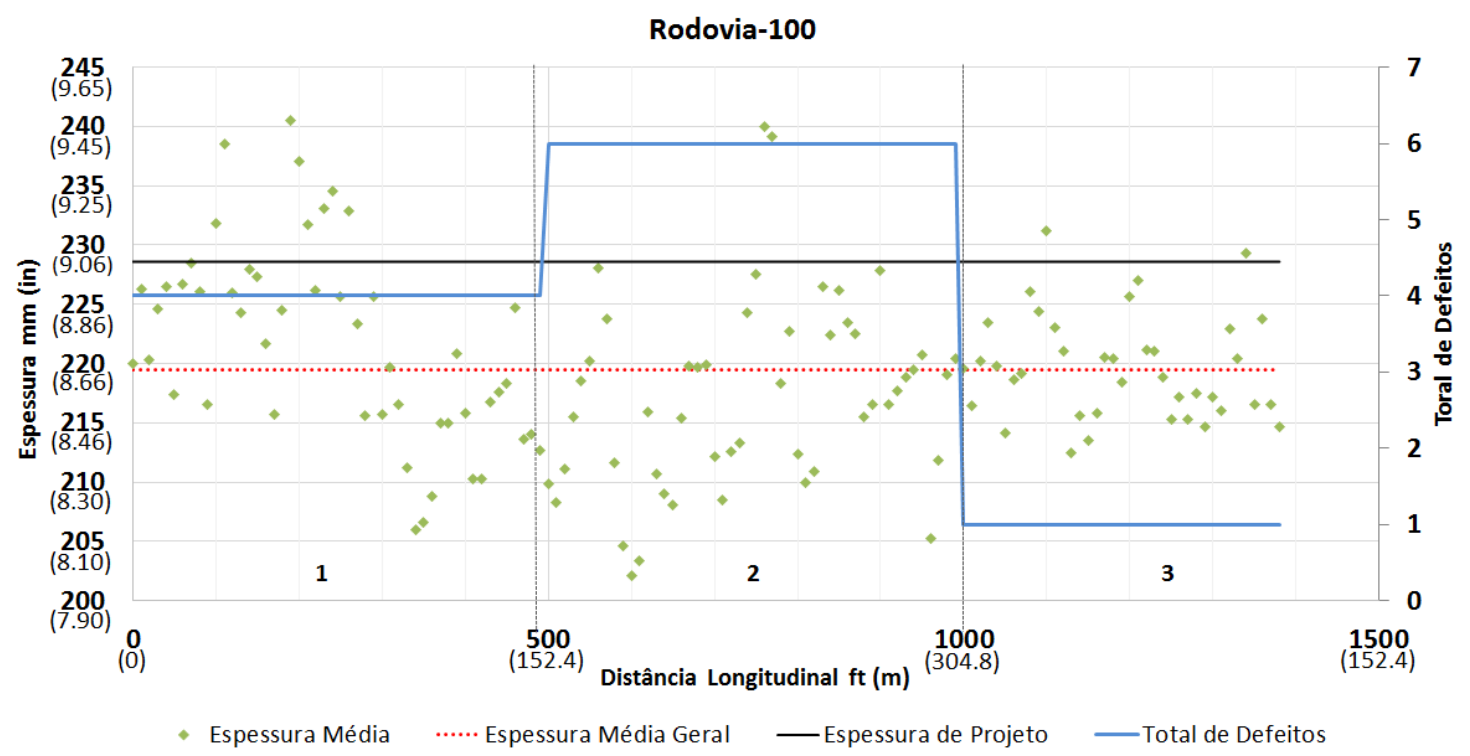

Figura 6: Variação longitudinal da espessura versus defeitos (Rodovia 100)

Nesta rodovia, observou-se uma considerável diferença entre a menor espessura observada e a espessura de projeto (diferença de aproximadamente $39,1 \mathrm{~mm}$ ), o que incorreria nas mesmas penalizações indicadas pelo MnDOT (2015) para a Rodovia 100. Notou-se também que a espessura média de todas as medidas $(219,5 \mathrm{~mm})$ foi inferior à espessura de projeto $(228,6$ $\mathrm{mm})$. Caso esta diferença $(9,1 \mathrm{~mm})$ tivesse sido observada pelo controle tecnológico indicado pelo mesmo órgão, a empresa responsável pela execução deveria ter a dedução no preço unitário pactuado de US\$0,90 por $\mathrm{m}^{2}$.

$\mathrm{Na}$ análise dos segmentos individualmente, todos os segmentos apresentaram espessuras médias próximas e ou inferiores à de projeto, com a maior média dada no segmento 1.0 segmento 3, mesmo tendo espessura média menor que a de projeto, apresentou um menor número de defeitos, o que demonstra um melhor desempenho em relação aos outros segmentos. Este comportamento não pode ser justificado pela espessura média já que ela é similar nos três segmentos, logo, pode-se aventar a correlação deste comportamento com o menor desvio padrão 
das medidas. Da mesma forma como observado na Rodovia 60, o melhor desempenho é obtido nos segmentos com menores desvios padrões, ainda que apresentem pequena deficiência de espessura. É importante notar que a diferença de desempenho dos segmentos 2 e 3 não pode ser atribuído à espessura da placa visto que os valores de espessura nos dois segmentos são semelhantes. Tal constatação contraria o senso comum, quando para semelhantes condições de tráfego e climática, placas com espessura inferior à de projeto apresentariam pior desempenho.

No estudo transversal da rodovia 100 (Tabela 4), relativo ao desvio padrão das medidas de espessura, a posição 5 obteve o maior valor e a posição 4 obteve o menor. Em relação à espessura média, o maior valor foi encontrado novamente na posição 5 e o menor na posição 1, assim sendo, a posição 5 é a que possui maiores desvio padrão e espessura média. Na comparação com a Rodovia 60, a posição 5 continua sendo a posição com maior desvio padrão e maior média de espessura.

\subsection{Interestadual 394}

Ao examinar a Figura 7, é possível observar a existência de um grande número de pontos acima da linha de espessura de projeto, fazendo com que a linha de espessura média ficasse acima da espessura de projeto. Quanto à dispersão de pontos, tem-se um menor desvio padrão se comparado com a Rodovia 100 e Rodovia 60, com desvio padrão geral do trecho de 8,3 mm.

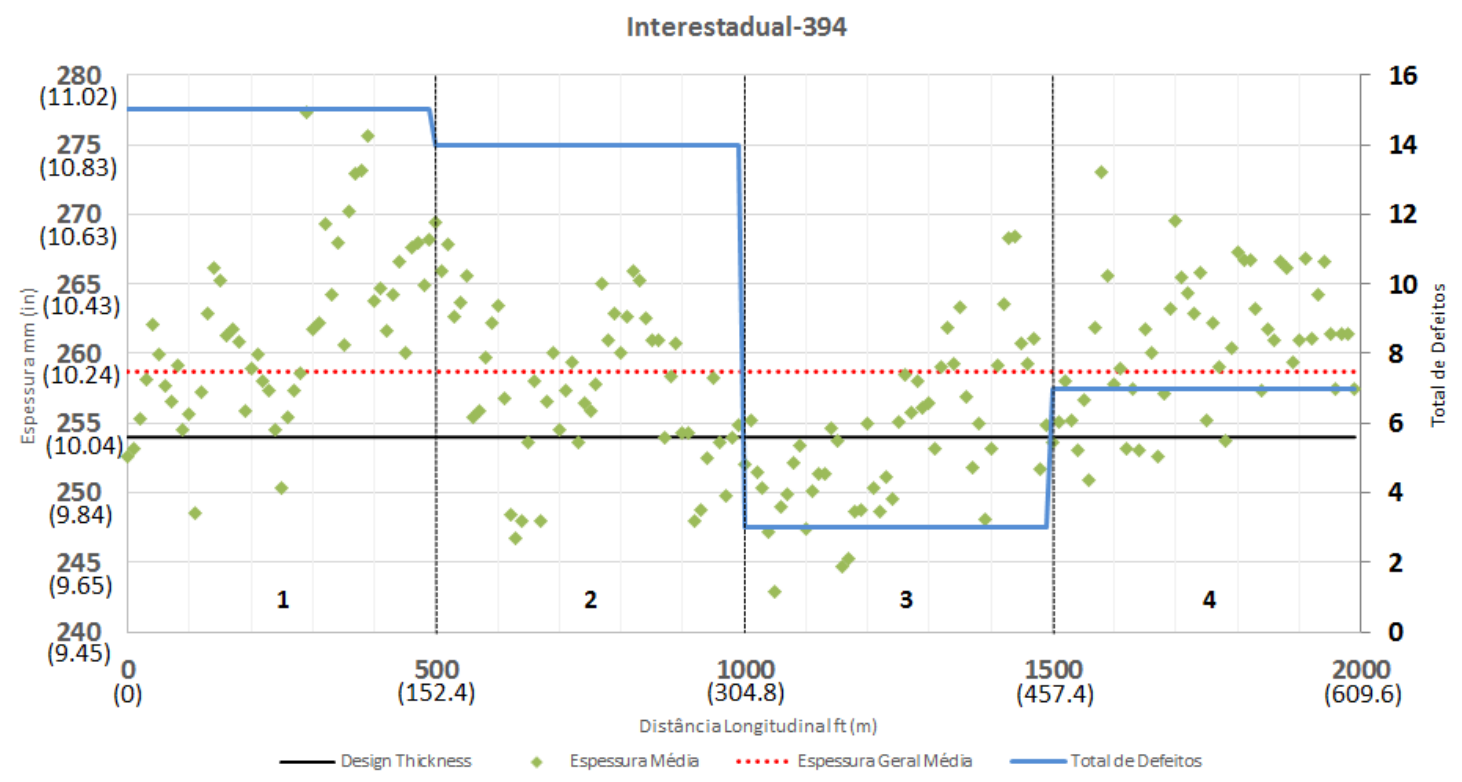

Figura 7: Variação longitudinal da espessura versus defeitos (Interestadual 394)

A diferença entre a espessura de projeto $(254 \mathrm{~mm})$ e o valor da espessura mínima (231,5 $\mathrm{mm}$ ) neste trecho foi de 22,5 mm. Caso estes valores fossem obtidos pela fiscalização, segundo critérios normativos previstos em MnDOT (2015), a área de abrangência da referida diferença de espessura, 22,5 mm, implicaria em uma das duas possibilidades, conforme decisão do órgão:

1. Remoção e substituição das placas naquela região, sem custos para o departamento; ou

2. empreiteiro receberia pelo serviço realizado mas com uma dedução do preço unitário do serviço de US $\$ 25,00 / \mathrm{m}^{2}$ relativo à região com diferença de espessura (projetada e executada) compreendida entre $13 \mathrm{~mm}$ e $25 \mathrm{~mm}$. 
Entretanto, comparando a Rodovia Interestadual 394 com os dois trechos previamente avaliados, este possui a menor variação de espessura. A diferença entre a espessura média (258,7 $\mathrm{mm}$ ) e a de projeto é de $4,7 \mathrm{~mm}$ e, caso esta diferença tivesse sido observada pelo controle tecnológico indicado pelo órgão, a empresa responsável pela execução deveria ter a dedução no preço unitário pactuado de US $\$ 0,25$ por $\mathrm{m}^{2}$.

$\mathrm{Na}$ análise individual de cada segmento, fica claro que todos possuem média similar ou maior que a espessura de projeto, além das médias individuais não apresentarem diferenças significativas como nas rodovias apresentadas anteriores. Na Interestadual 394, foram identificados os maiores valores de desvio padrão as seções 1 e 2 (respectivamente 7,8 e 8,1 mm), os menores são das seções 3 e 4 (respectivamente 7,6 e 7,4 mm). Assim, da mesma forma como fora evidenciado anteriormente, as seções 1 e 2 com maior desvio padrão apresentam maior número de defeitos, enquanto que nas seções 3 e 4 com menores valores de desvio padrão exibem menor número de defeitos (Figura 7).

Processando os dados em relação às posições transversais, tem-se a posição 1 e 5 os maiores valores de desvios padrões e a menor foi encontrado na posição 3, em contrapartida, a posições que obtiveram as maiores médias fora a 3 e 5, sendo a 1 é a que obteve menor. Tendência de distribuição de maiores espessuras na borda longitudinal interna das placas também identificada nas outras duas rodovias avaliadas neste trabalho.

\subsection{Análise Geral}

Nas três rodovias analisadas neste artigo, notou-se três relações diferentes entre espessuras médias aferidas e de projeto. A Rodovia 100 apresentou espessura média menor que a de projeto, por outro lado, a Interestadual 394 tem média maior do que a de projeto; já a Rodovia 60 exibe a terceira distribuição possível, na qual a espessura média é similar à de projeto, como mostra a Figura 8. Estas três combinações, completamente diferentes, assim como a elevada amostragem de dados extraídos em campo garantiram a confiabilidade das análises realizadas neste estudo.

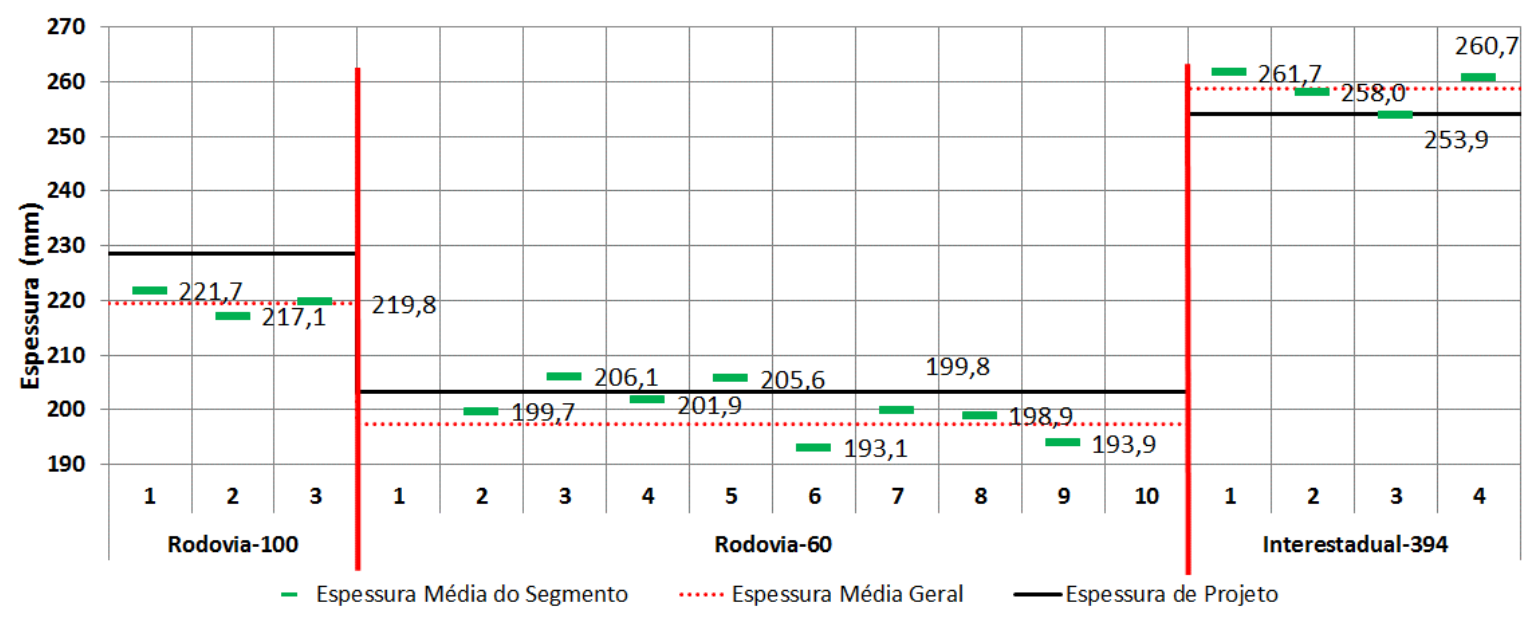

Figura 8: Distribuição de espessuras

O senso comum, conforme já mencionado, indicaria que o desempenho do pavimento é altamente dependente da relação entre a espessura real da placa de CCP e a de projeto. Todavia, esse estudo sugere existir um outro parâmetro de relevância para o desempenho do pavimento: 
o desvio padrão da espessura. A correlação entre este importante parâmetro e o desempenho do pavimento é evidente em todas as três vias analisadas. Segmentos com maior desvio padrão da espessura apresentaram o maior número de defeitos. Nas Figuras 9 (b, c e d), há uma clara tendência (apesar do baixo índice de correlação) de que quando se aumenta o desvio padrão da espessura, aumenta-se o número de defeitos. Em relação às três rodovias avaliadas com diferentes parâmetros de projeto, o mesmo comportamento foi observado ao plotar os resultados conjuntamente, como demonstra a Figura 9a.

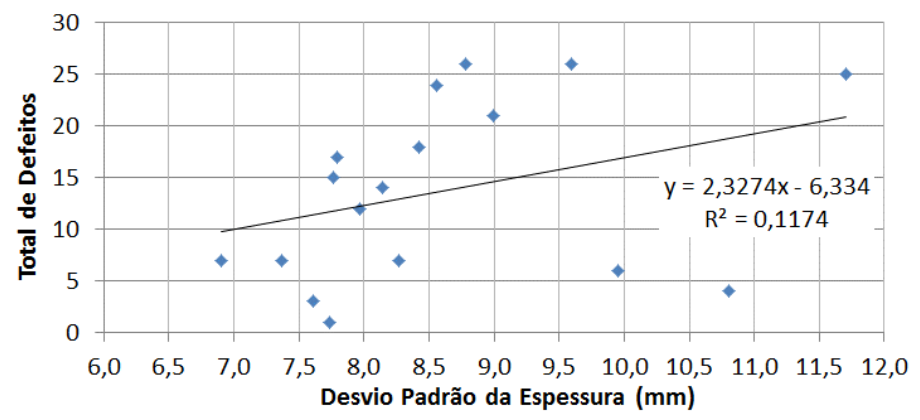

(a)

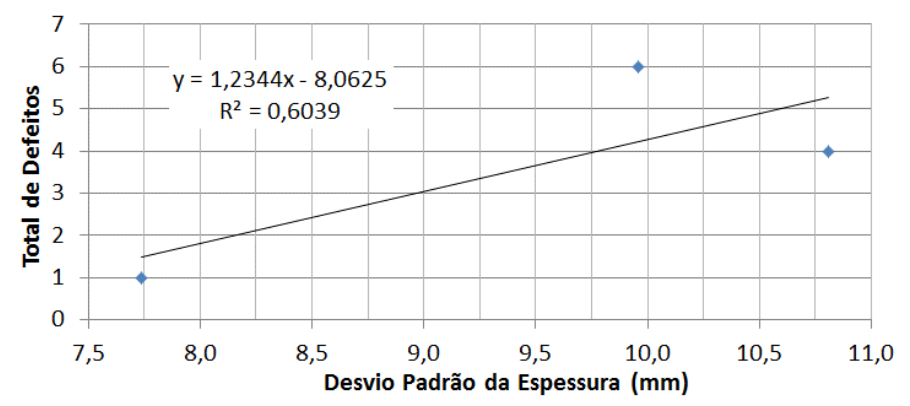

(b)

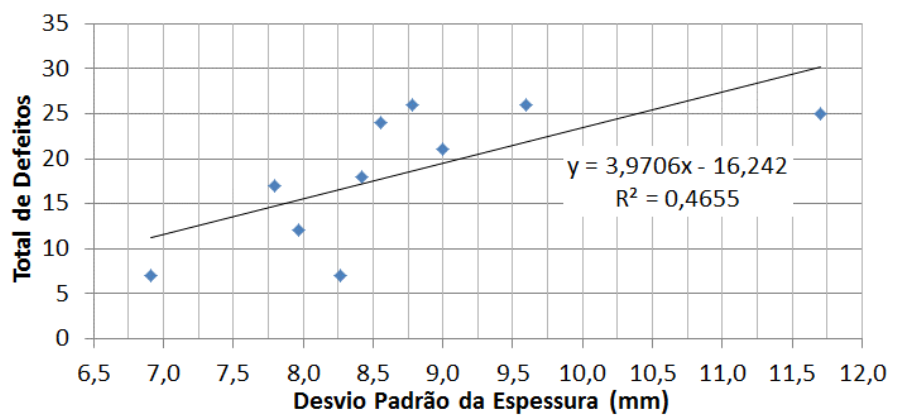

(c)

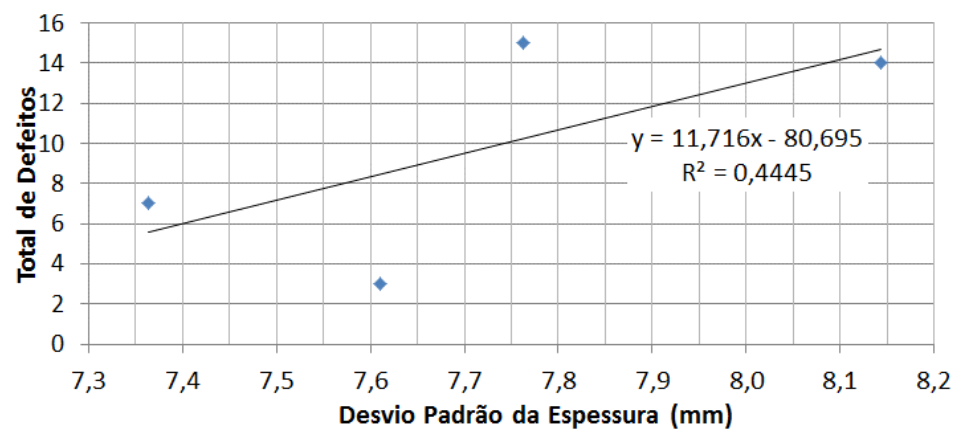

(d)

Figura 9: Desvio padrão da espessura versus número de defeitos para (a) todas as seções; (b) Rod-100; (c) Rod-60; e (d) I-394 
No tocante à variação da espessura na direção transversal do pavimento, a menor espessura média está sempre localizada na posição 1 (próxima ao acostamento) e, na maioria dos casos, a maior espessura média e, também, o maior desvio padrão estão localizados na posição 5. Portanto, considerando que nas posições 1 e 5 foram avaliadas as maiores variações de espessuras, recomendar-se-ia que os testes de controle de espessuras fossem conduzidos, prioritariamente, nestas posições.

Outro detalhe relevante sedia-se no fato das simulações numéricas a partir de elementos finitos indicarem que a posição crítica de carregamento em uma placa de concreto com barras de transferência estar situada na junta longitudinal externa da placa, exatamente a posição 1, localização esta de menor espessura avaliada neste estudo. Esta constatação poderá, em casos mais severos e específicos, contribuir para falhas estruturais precoces dos pavimentos de CCP, conforme já demonstravam estudos clássicos, como Packard e Tayabji (1985).

Os resultados demonstram que pequenas variações de espessura acima ou abaixo da espessura de projeto são insignificantes para o desempenho do pavimento, aferidos neste trabalho pelo número de defeitos. Na realidade, observou-se que a uniformidade de execução das placas, ou seja, espessura uniforme ao longo da rodovia, está intimamente relacionada ao desempenho do pavimento. Esta constatação, em termos práticos de engenharia, reveste-se de peculiar importância, uma vez que muitos departamentos de transporte (nacionais e internacionais) focam suas observações, quase que, exclusivamente, sobre o controle da espessura mínima de projeto, sem verificar sua dispersão a partir de um significativo número de amostras (demandaria grande número de extrações de testemunhos).

Segmentos que seriam penalizados financeiramente pela forma de condução do controle tecnológico de pavimentos de concreto do MnDOT, por exemplo, em função da espessura deficiente, apresentaram desempenho melhor do que segmentos onde a espessura das placas de concreto estavam acima daquela projetada, conforme visto neste estudo.

\section{CONCLUSÃO}

Este artigo analisou a variação de espessura longitudinal e transversal obtida através de teste não destrutivo com aparelho ultrassônico em três rodovias com placas de concreto nos Estados Unidos. A correlação da variação de espessura com os defeitos observados através de software de imagem na superfície do pavimento permitiu elencar as seguintes conclusões:

i. desempenho do pavimento de concreto, baseado, neste estudo, pelo número de defeitos, não é unicamente dependente da relação entre a real espessura em campo e a de projeto. Placas com espessuras ligeiramente abaixo daquela dimensionada não apresentaram um maior número de defeitos, desde que apresentassem baixo desvio padrão de espessura de placa;

ii. desvio padrão da espessura pode ser considerado um parâmetro importante para o desempenho do pavimento, pois traz relevantes informações sobre a uniformidade construtiva;

iii. A elevada densidade de pontos amostrados pelo método ultrassônico indica que empreiteiros e departamentos de transporte poderiam focar seus esforços em espessuras uniformes em ambas as direções, principalmente nas proximidades das bordas longitudinais internas e externas da placa, onde as espessuras encontram valores máximos e mínimos. 
iv. Senso comum de que espessuras de placa acima da projetada resultam em melhor desempenho não foi observado por este estudo;

v. Os resultados também possuem implicações importantes em relação ao erário público e/ou privado, assim como nos quesitos de sustentabilidade uma vez que espessuras além da projetada não implicará, necessariamente, em melhor desempenho dos pavimentos de CCP.

\section{REFERÊNCIAS}

DNIT (2004) Pavimento Rígido - Execução de pavimento rígido com equipamento de fôrmas-deslizante - Especificação de serviço. DNIT 049/2004-ES. Departamento Nacional de Infraestrutura de Transportes.

EDWARDS, L (2012) Evaluation of Technologies for Nondestructively Determining Concrete Pavement Thickness. Proceedings of the $10^{\text {th }}$ International Conference on Concrete Pavement, ISCP, Québec City, Canada.

FHWA (2003). Distress Identification Manual for Long-Term Pavement Performance Program, FHWA-RD-03-031. US Department of Transportation, Federal Highway Administration.

HOEGH, K.; KHAZANOVICH, L.; YU, T (2011) Ultrasonic Tomography for Evaluation of Concrete Pavements. Transportation Research Record, v. 2232, p. 85 - 94. DOI: 10.3141/2232-09

HOEGH, K.; KHAZANOVICH, L.; YU, T. (2012). Concrete Pavement Joint Diagnostics with Ultrasonic Tomography. Transportation Research Record, v. 2305, p. 54 - 61. DOI: 10.3141/2305-06

HOEGH, K.; KHAZANOVICH, L.; WOREL, B. J.; YU, T. (2013). Detection of Subsurface Joint Deterioration Blind Test Comparison of Ultrasound Array Technology with Conventional Nondestructive Methods. Transportation Research Record, v. 2367, p. 3 - 12. DOI: 10.3141/2367-01

JIANG, Y., SELEZNEVA, O., MLADENOVIC, G., AREF, S., and DARTER, M (2003). Estimation of Pavement Layer Thickness Variability for Reliability-Based Design. Transportation Research Record. 1849, pp. 156-65. DOI: 10.3141/1849-17

KIM, S. M., MCCULLOUGH, B. F (2002). Reconsideration of Thickness Tolerance for Concrete Pavements. Report No. 4382-1, Federal Highway Administration, Washington, DC.

MnDOT (2011). Pavement Distress Identification Manual. Minnesota: Department of Transportation, St. Paul.

MNDOT (2015). Standard Specifications for Construction: 2016 Edition. Minnesota: Department of Transportation, St. Paul.

MnDOT (2006). An Overview of Mn/DOT's Pavement Condition Rating Procedures and Indices. Minnesota: Department of Transportation St. Paul.

NCHRP (2004). Guide for Mechanistic-Empirical Design of new and rehabilitated pavement structures, final report, part 3. Design Analysis, chapter 4. Design of new and reconstructed rigid pavements, Illinois, ERES Consultants Division.

PACKARD, R. G. e TAYABJI, S. D (1985). New PCA thickness design procedure for concrete highway and street pavements. 3th International Conference on Concrete Pavement Desgin. Proceedings. Purdue University. pp. 225-236. West Lafayette.

SALLES, L. S., BALBO, J. T. e KHAZANOVICH, L. Non-destructive ultrasonic tomography for concrete pavement evaluation: signal processing and image analysis of crucial parameters. Revista Ibracon de Estruturas e Materiais, v. 10, p. 1182-1191, 2017. DOI: $10.1590 / \mathrm{s} 1983-41952017000600004$

STUBSTAD, R. N., TAYABJI, S. D., LUKANEN, E. O. (2002). LTPP Data Analysis: Variations in Pavement Design Inputs. Report No. Project 20-50 [5]), National Cooperative Highway Research Program Transportation Research Board National Research Council, Washington, DC.

VANCURA, M., KHAZANOVICH, L., BARNES, R (2013). Variation Assessment with Cores and Nondestructive Testing Measurements. Transportation Research Record, v. 2347, p. 61 - 68. DOI: 10.3141/2347-07 